\title{
Mood Sensing using Facial Landmarks
}

\author{
Kuppala Sushmanth Sai, Yaramala Srinath, P.Sabitha, Bejawada Venkatesh, Raghavendran R
}

\begin{abstract}
Communication plays a pivotal role in every person's life.There are various types of communications in which some are verbal and some are non-verbal. Expressions on a person's face are a type of non-verbal communication.Expressions on the face can be used to define how the person is feeling, recognizing them helps to enhance the human-machine interaction.Thus we propose a system that is un-affected by the illumination changes or the light changes. Expressions on the human face can be computed by using CLM,constrained local models inserts a dense model to a new input image to get the emotions stats.SVM classifier is used to distinguish the input image into different emotion categories. Results showed a remarkable increase in efficiency and performance. Change in lighting conditions will have a very little effect on the efficiency of the system.
\end{abstract}

Keywords: SVM, MEM, CLM

\section{INTRODUCTION}

Due to increasing technological inventions in the digital world,the need for a better security against tech thefts has increased enormously therefore the human face serves as a significant tool to distinguish a person from another. Human face is a crucial biometric object in image and video databases of police investigation systems.Face recognition contains a important role in biometric systems and is enticing for various applications together with visual police investigation and security.Due to the wide acceptance all over the world about the expression recognition systems, it can truly become the new face of firewalls against the cyber thefts.Face pictures also are the sole biometric data on the market in some national databases and international terrorist watch-lists and might be nonheritable even while not subject's cooperation.

In recent years efforts have been made to improve and develop an error-free emotion detection system, but many obstacles lie in the path of efficiency like outdoor illumination,lower image quality,occlusion,blurred image etc.Therefore emotion detection has become a difficult task

Revised Version Manuscript Received on July 20, 2019.

Kuppala Sushmanth Sai,: Srm University Ramapuram,Chennai in Computer Science

Yaramala Srinath, Srm University Ramapuram,Chennai in Computer Science

P. Sabitha, M.E., Assistant Professor, Department of Computer Science and Engineering, Srm Institute of science and technology.

Bejawada Venkatesh: Srm University Ramapuram,Chennai in Computer Science Science

Raghavendran, Srm University Ramapuram,Chennai in Computer to achieve.Emotion detection systems have a wide range of applications in the modern world in both private and public sector.The key factor in determining an industry's success is the customer satisfaction.It forms one of the primary goals of the industry but there are very few emotion detection methods without direct communication to understand how the customer is feeling about the product.Along with that an application which can capture the mood of the students during classes helps the lecturer to assess the effectiveness of the lecture doesn't exist.Therefore an efficient and dependent expression detection system is the need of the hour.

The proposed system is based on computer vision technology where the facial landmarks are recognized using CLM(constrained local model) and expression is categorized using SVM Classifier.Pandas is used for pre-processing.Tensorflow is used for manipulating deep learning models using python. The distance between the facial landmarks is calculated by using Euclidean formula and the expression is labelled based on the distance.

\section{A. Related Work}

1.The paper delivered by Gozde Yolcu, Ismail Oztel, Serap Kazan in the year 2017 presents a novel profound learning approach for programmed outward appearance recognition. The arranged structure first portions the facial components renowned to be fundamental for face acknowledgment and structures an iconized picture at that point performs face order abuse the acquired iconized facial parts picture joined with the crude facial images.[2]Impaired outward appearance is a typical indication of various ailments. Transcendent models run from adolescence neurodevelopmental clutters For the majority of these neurologic conditions, clinical conclusion and illness checking after some time can be very testing, regularly requiring medicinal testing that is intrusive as well as expensive.Thus, there is a basic need to create noninvasive, minimal effort choices.

2.The paper created by Su-Jing Wang,Wen-Jing Yan Xiaobai Li, Guoying Zhao,Chun-Guang Zhou, Xiaolan Fu in the year 2015 uncovers that two perceptual shading spaces (CIELab and CIELuv) give valuable data to articulation recognition,along with Tensor Independent Color Space (TICS), to help perceive miniaturized scale articulations. In this paper, it is demonstrated that CIELab and CIELuv are additionally useful [3] Micro-articulations are brief outward appearances that uncover the feelings that an individual attempts to disguise, particularly in high-stakes circumstances Although small scale articulations have potential application in an assortment of

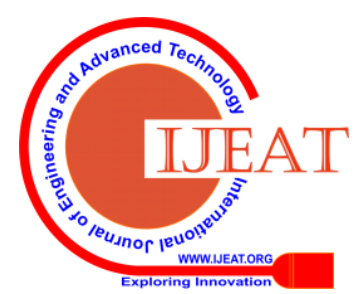




\section{Mood Sensing using Facial Landmarks}

fields, people experience issues in identifying and perceiving them. Thus there is a need to recognize them carefully.

3.The paper created by Washef Ahmed,Soma Mitra,Kunal Chanda ,Debasis Mazumdar in the year 2013.This paper displays improving the acknowledgment precision of at least one of the six first articulations especially bliss, shock, dread, nauseate, bitterness and outrage from the blend of two facial expressions.For this reason a movement inclination based optical stream for muscle development is figured between edges [4]The number of youngsters determined to have mental imbalance has mushroomed over the most recent couple of years. People with chemical imbalance are accepted to have a broken engine neuron framework. For therapists and social researchers, outward appearance examination and their measurement has been a functioning exploration point since the original work of Darwin. Ekman announced their outcome on classification of human outward appearances as joy, shock, dread, sicken, bitterness and outrage.

4.The paper created by S. Ramanathan ,Ashraf Kassim ,Y.V. Venkatesh,Wu Sin Wah in the year 2006 presents a novel way to deal with the recognition and arrangement of human outward appearances utilizing a morphable 3D model.The different looks of a private utilizing a face scanner that produces harsh finished 3D networks abuse stereoscopic reconstruction.A Morphable Expression Model (MEM), that joins feeling dependentface varieties as far as transforming parameters, is then processed by setting up correspondence among the emotive countenances. [4] Despite noteworthy advancement in picture based face and demeanor acknowledgment, no mechanized framework has been appeared to work tastefully outside a controlled environment.Since the face picture is a functionof enlightenment, perspective, present, shading and so on., acknowledgment has ended up being troublesome. As the face is a lively $3 \mathrm{D}$ object, shape data seems more qualified to portray the face than its projection as a $2 \mathrm{D}$ force image.Also, the $3 \mathrm{D}$ face structure is unfeeling to cause, brightening or shading; and acknowledgment rates are found to broaden extensively once structure information is joined with dim dimension information.

\section{METHODOLOGY}

SVM CLASSIFIER:SVM is a gathering of learning calculations basically utilized for arrangement assignments on convoluted information, for example, picture grouping and protein structure examination. SVM is utilized in an incalculable fields in science and industry, including Bio-innovation, Medicine, Chemistry and Computer Science.

It has conjointly dressed to be in a perfect world fit to order enormous content vaults like those housed in about all substantial, present day organizations.In specific its capacity to catch patterns decided in an almost no instructing set and to sum up those patterns against a more extensive corpus have made it accommodating over a curiously large number of applications.SVM utilizes an administered learning approach, which infers it figures out how to group inconspicuous data bolstered an accumulation of named instructing data, for example, corporate documents. The introductory arrangement of preparing information is

commonly recognized by space specialists and is utilized to assemble a model that can be connected to some other information outside the preparation set.The exertion expected to develop a top quality training set is kind of humble, essentially contrasted with the level of information which will be eventually characterized against it.This implies that learning calculations like SVM give partner extraordinarily cost compelling system of content characterization for the huge volumes of archives made by in vogue organizations. The equalization of this paper covers the inward operations of SVM, its application in science and business, the legitimate solidness of the strategy just as arrangement precision contrasted with manual classification.SVM is most customarily acclimated part one information set of reports into 2 particular subsets.

The SVM equation figures out how to differentiate between the 2 classes upheld an instructing set of reports that contains marked precedents from every class.

Euclidean formula is used to find out the distance between two data points and categorise the facial feature accordingly. The Euclidean distance between points $\mathrm{p}$ and $\mathrm{q}$ is the length of the line phase connecting them.In Cartesian coordinates, if $\mathrm{p}=(\mathrm{p} 1, \mathrm{p} 2, \ldots, \mathrm{pn})$ and $\mathrm{q}=(\mathrm{q} 1, \mathrm{q} 2, \ldots, \mathrm{qn})$ are two points in Euclidean n-space, then the distance (d) from $p$ to $\mathrm{q}$, or from $\mathrm{Q}$ to $\mathrm{p}$ is given by the formula.

$$
\begin{aligned}
& d(p, q)=d(q, p) \\
& =\sqrt{\left(q_{1}-p_{1}\right)^{2}+\left(q_{3}-p_{3}\right)^{2}+\cdots+\left(q_{n}-p_{n}\right)^{2}} \\
& =\sqrt{\sum_{i=1}^{n}\left(q_{i}-p_{i}\right)^{2}}
\end{aligned}
$$

III . SYSTEM ARCHITECTURE

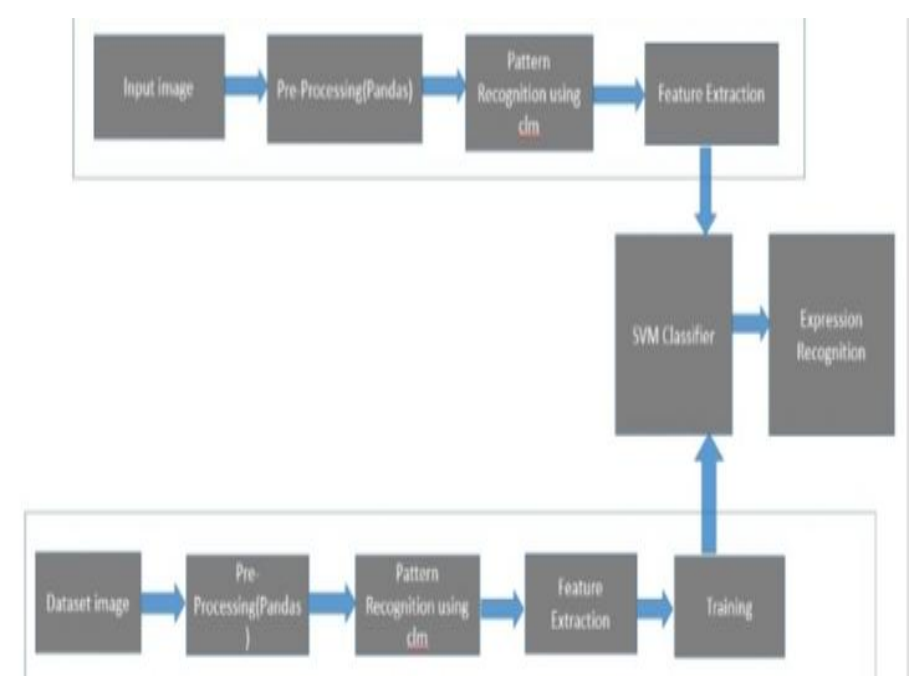

Fig.

Architecture Diagram

1.

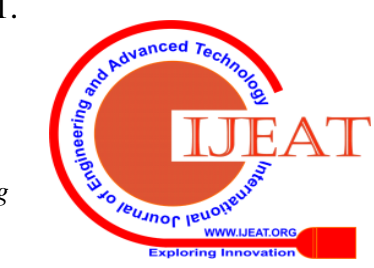



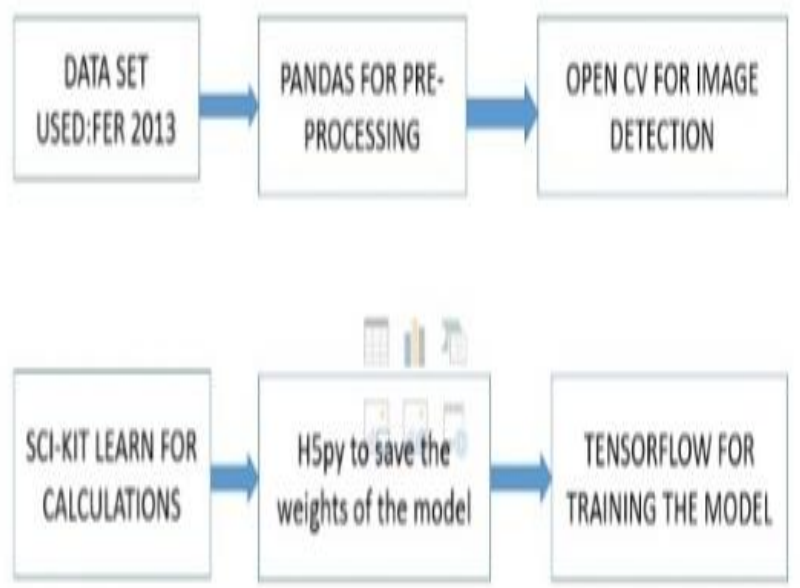

Fig.2.Software

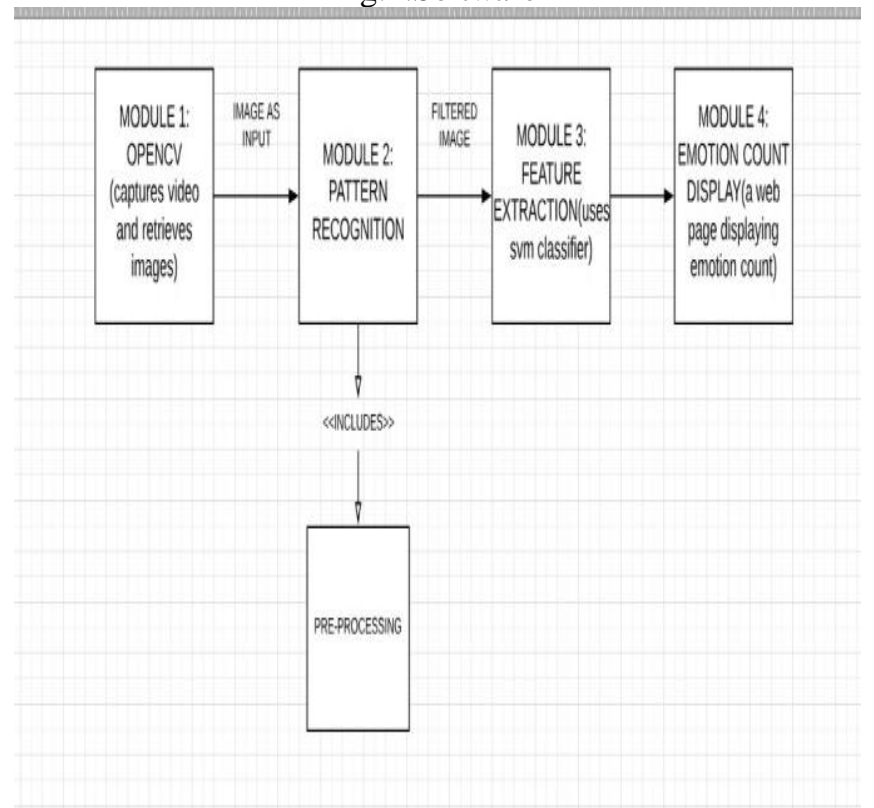

Requirements
Digital Processing: The distinguishing proof of articles in a picture would presumably begin with picture preparing procedures, for example, commotion evacuation, trailed by (low-level) highlight extraction to find lines, districts and potentially regions with certain textures. The input picture is decreased to dim scale picture. A grayscale Image is advanced picture is an image amid which the value of each constituent might be a solitary example, that is, it conveys just force data. Grayscale pictures have a few update dim in the middle.

\section{MODULE DESCRIPTION}

Module 1-OPENcv: In this project computer vision is utilized to recreate, interfere, and comprehend a 3D scene from its $2 \mathrm{D}$ pictures, as far as the properties of the structure present in the scene. It manages demonstrating and duplicating human vision utilizing computer programming and hardware. A continuous module which can catch client's face through webcam steaming utilizing Open $\mathrm{CV}$, which crops the face and recognizes from the first casings and the edited pictures are resized to $48 \times 48$ grayscale picture, at that point accept them as contributions for the profound learning models.

Module 2-Feature Extraction:An input image is first preprocessed using pandas. The point of pre-processing is to improve the picture information that smothers undesirable contortions or upgrades some picture highlights vital for further processing. Such clamor decrease will be an ordinary pre-preparing venture to upgrade the consequences of later procedure.

Module 3-Pattern Recognition : Data of the human face can be figured by methods for obliged local models (CLM), which fits a thick model to an inconspicuous picture in an iterative way. We utilized a SVM classifier to arrange the procured picture into various feeling classifications.

MODULE 4:Emotion Count Display:In this module the output or the results of the system are displayed which is nothing but the percentage of emotions that are being displayed over a period of time in front of the web-cam.Along with that a graph is used to display the ratio of each emotion that is being displayed over the course of time which helps to understand the trend of each emotion during the whole time period

Fig:3 UML Representation of modules

\section{V:RESULTS}

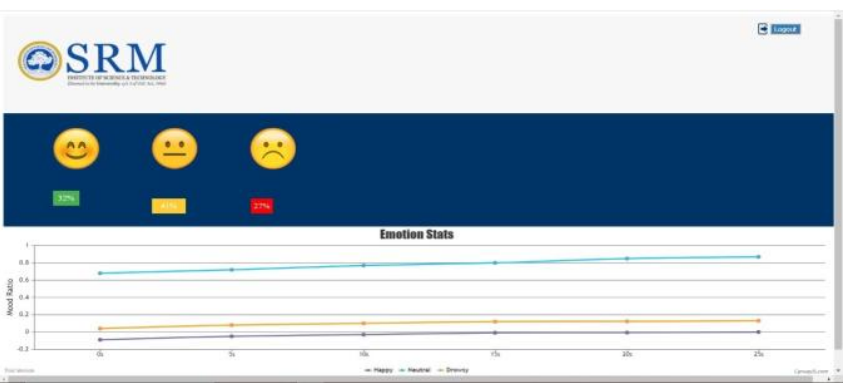

Fig:4 Emotion Count Display

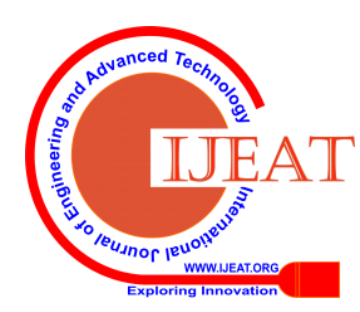




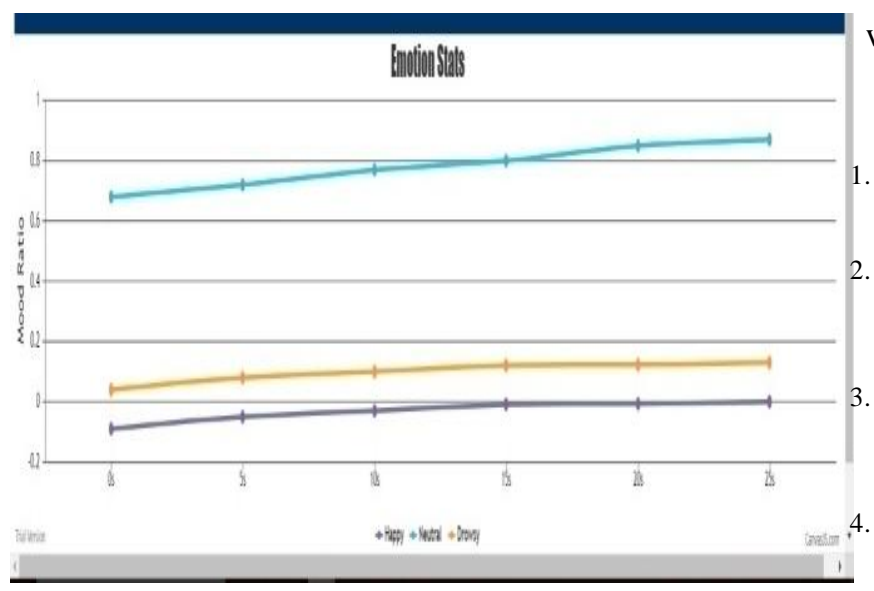

Overall the model has an accuracy that is higher than the existing models.

6 emotions- Angry, Sad, Neutral, Drowsy, Surprise, Happy can be detected.Where sad,surprise are listed under neutral category for understanding purposes.

\section{CONCLUSION}

Automated identification of facial expressions may reveal clinically relevant facial expression features that can distinguish between the different moods of a person and also aid in determining whether a person is interested in doing that work or not .Example :A teacher can find out whether the students are being attentive or feeling drowsy with the help of this application.

\section{FUTURE ENHANCEMENT}

Automated expression recognition can be used in defence sector by implementing additional security parameters like retina recognition or fingerprint recognition, thus the system can serve as a firewall against intruder invasions..

\section{ACKNOWLEDGMENT}

We place on regard of our deep sense of gratitude to our lionized Chairman Dr.R.SHIVAKUMAR for providing us with the requisite infrastructure throughout the course.

We take the opportunity to extend our hearty and sincere thanks to our Dean, Dr.V.SUBBIAH BHARATHI, M.Tech, $\mathrm{Ph} . \mathrm{D}$., for maneuvering us into accomplishing the project.

We take the privilege to extend our hearty and sincere gratitude to the Professor and Head of the Department, Dr.J.JAGADEESAN, M.Tech., Ph.D., for his suggestions, support and encouragement towards the completion of the project with perfection.

We convey our hearty and sincere thanks to our Project Coordinator Mr. S.ARUN KUMAR, M.Tech., for his fortification. We express our hearty and sincere thanks to our guide Mrs. P Sabitha, M.E., Assistant Professor, Computer Science and Engineering Department for her sustained encouragement, consecutive criticism and constant guidance throughout this project work.

Our thanks to the teaching and non-teaching staff of the Computer Science and Engineering department of SRM Institute of Science and Technology, Ramapuram Campus,

who provided necessary resources for our project.

\section{REFERENCES}

Kaihao Zhang, Yongzhen Huang "Facial Expression Recognition Based on Deep Evolutional Spatial-Temporal Networks",IEEE Transactions on Image processing 2017.

Gozde Yolcu, Ismail Oztel, Serap Kazan, Cemil Oz, Kannappan Palaniappan, Teresa E. Lever, Filiz Bunyak "Deep Learning-based Facial Expression Recognition for Monitoring Neurological Disorders"IEEE international conference on bio informatics"

Su-Jing Wang ; Wen-Jing Yan ; Xiaobai Li ; Guoying Zhao ; Chun-Guang Zhou ; Xiaolan Fu ; Minghao Yang ; Jianhua Tao, "Micro-Expression Recognition Using Color Spaces, IEEE Transactions Image Processing 2015

Washef Ahmed ; Soma Mitra ; Kunal Chanda ; Debasis Mazumdar "Assisting the autistic with improved facial expression recognition from mixed expressions" IEEE international conference on computer vision 2013"

5. S. Ramanathan ; Ashraf Kassim ; Y.V. Venkatesh ; Wu Sin Wah "Human Facial Expression Recognition using a 3D Morphable Model" IEEE conference on Image Processing 2006.

6. M. Pantic ; I. Patras"Dynamics of facial expression: recognition of facial actions and their temporal segments from face profile image sequences" IEEE Transactions on Systems, Man, and Cybernetics, Part B (Cybernetics) 2006.

\section{AUTHORS PROFILE}

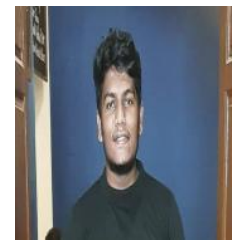

Mr.Kuppala Sushmanth Sai,:He has done his graduation in Srm university ramapuram,Chennai in computer science branch and has previously published a paper on alternate route traffic display system using dijkstra's algorithm.He is currently working for a multi national company as software developer.He has Undergone various courses on artificial intelligence and machine learning.

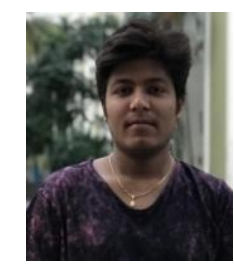

learning.
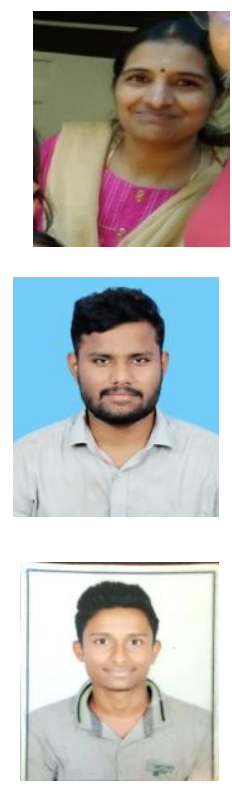

Mr.Yaramala Srinath, $\mathrm{He}$ has done his graduation in Srm university ramapuram,Chennai in computer science branch and has previously published a paper on alternate route traffic display system using dijkstra's algorithm.He is currently looking to do his masters abroad and specialistation in computer science.He has undergone various courses on artificial intelligence and machine

Mrs. P.Sabitha, M.E., Assistant Professor, Department of Computer Science and Engineering,Srm Institute of science and technology.She has done her masters degree on engineering and currently working as a computer science professor in srm university ramapuram Chennai

Bejawada Venkatesh:He has done his graduation in Srm university ramapuram, Chennai in computer science branch and has previously published a paper on alternate route traffic display system using dijkstra's algorithm.He is currently working for a multi national company as softwardeveloper.Hehas undergonevarious courses on artificial intelligence and machine learning.

Mr.Raghavendran, He has done his graduation in Srm university ramapuram,Chennai in computer science branch and has previously published a paper on alternate route traffic display system using dijkstra's algorithm.He is currently working for a multi national company as software developer.He has Under various courses on artificial intelligence and machine learning. 